\title{
Novel Pathogenic Variant of X-Linked Adrenoleukodystrophy in Mother of Affected Boys: Better Late Than Never
}

\author{
Chakshu Chaudhry, Inusha Panigrahi, Priyanka Srivastava
}

Genetic \& Metabolic Unit, Department of Pediatrics, Post Graduate Institute of Medical Education and Research (PGIMER), Chandigarh, India.

\section{Corresponding Author:}

Dr Priyanka Srivastava

Email: srivastavapriy@gmail.com

This is an Open Access article distributed under the terms of the Creative Commons Attribution License (creativecommons.org/ licenses/by/3.0).

Received : July 14,2020

Accepted : August 31, 2020

Published : October 5, 2020

\begin{abstract}
Background: X-linked adrenoleukodystrophy (XALD, OMIM\#300100) is the most common peroxisomal biogenesis disorder (PBD) presenting in the late childhood to adolescent age with neurological deterioration and diffuse central nervous system involvement. Case Report: We report a family which came to us for genetic counselling. Their two sons had progressive neuroregression and had expired in childhood. Molecular diagnosis confirmed by testing the mother by targeted next generation sequencing (NGS), with finding of a novel pathogenic variant in ABCD1 (OMIM*300371) gene. This variant resulted in a truncated protein which may lead to defects in very long chain fatty acids degradation. Genetic counselling was done, recurrence risk of the disease was explained and availability of prenatal testing was discussed. Conclusion: In certain cases of genetic disorder, where the DNA of the patient is not available, parental testing can be useful.
\end{abstract}

Keywords: Adrenoleukodystrophy, DNA, Genetic Counseling, Peroxisomal Disorders, Pregnancy.

\section{Introduction}

X-linked adrenoleukodystrophy (XALD, OMIM\#300100) is the most common peroxisomal biogenesis defect with a prevalence of 1 in 17,000 [1-3]. The childhood form presents in the late childhood to adolescents (5-15 years) with neurological deterioration and diffuse central nervous system involvement. It is an X linked disorder characterized by mutation in ABCD1 (OMIM*300371) gene. A couple came to us for pre-conceptional genetic counselling. Their two sons had similar course and expired. Not only was a molecular diagnosis confirmed by testing the mother, we also found a novel pathogenic variant in ABCD1 gene. With early referral and diagnosis of such patients, genetic counselling of the family can be offered timely.

\section{Case Report}

The propositus presented with behavioral problems like inattention, hyperactivity and poor school performance noted from 8-years of age. $\mathrm{He}$ was born of non-consanguineous marriage. There was no history of associated fever, abnormal body movements, organomegaly or exposure to toxins. He was shown at 10 -year age and computed tomography of the brain done showed periventricular white matter hypodensities with posterior periventricular foci of calcification and asymmetry of lateral ventricles. His birth and postnatal period were uneventful and there was no history of acute insult requiring hospital admission in the past. Keeping a possibility of X-linked adrenoleukodystrophy, VLCFA (very long chain fatty acids) analysis was performed by capillary 
gas chromatography/mass spectrometry. It showed C26:0 level of $1.080 \mathrm{microgram} / \mathrm{mL}$ (normal 0.23+ 0.09), and increased C24/C22 and C26/C22, values of $1.860(\mathrm{~N}-0.84+0.10)$ and $0.099(\mathrm{~N}-0.01+0.004)$ respectively. This was consistent with peroxisomal fatty acid oxidation defect, like X-linked adrenoleukodystrophy. The younger male sibling also had similar manifestations. Both children had progressive neuro-deterioration and died by the age of 11 years. Genetic counselling of parents was done. They were informed regarding availability of prenatal diagnosis and pre-implantation genetic diagnosis and in-vitro fertilization (IVF) to prevent having yet another affected child.

Mutation analysis was done in the mother by targeted next generation sequencing (NGS). A novel heterozygous variant c.617_618delinsT was identified in exon 1 of the ABCD1 gene (ABCD1; OMIM* 300371) (chrX:152991338 CC>T; Depth: 241x) [Fig.1a] that resulted in frameshift mutation at codon 206 (Ala206ValfsTer10). This variant resulted in a shorter truncated protein $(\sim 214$ AA) as compared to reference protein (745AA) [Fig.1b]. The p.Ala206ValfsTer10 variant has not been reported in the 1000 genomes, ExAC, ClinVar, HGMD and ALD Mutation Database. The in silico prediction of the variant is damaging by MutationTaster2. The reference codon is conserved across species. This variant is located in the transmembrane domain of ALDP which is a region critical for protein structure and function. The variant was confirmed by Sanger sequencing [Fig.1c]. It has been submitted in ClinVar database (Accession ID is VCV000870373.1).

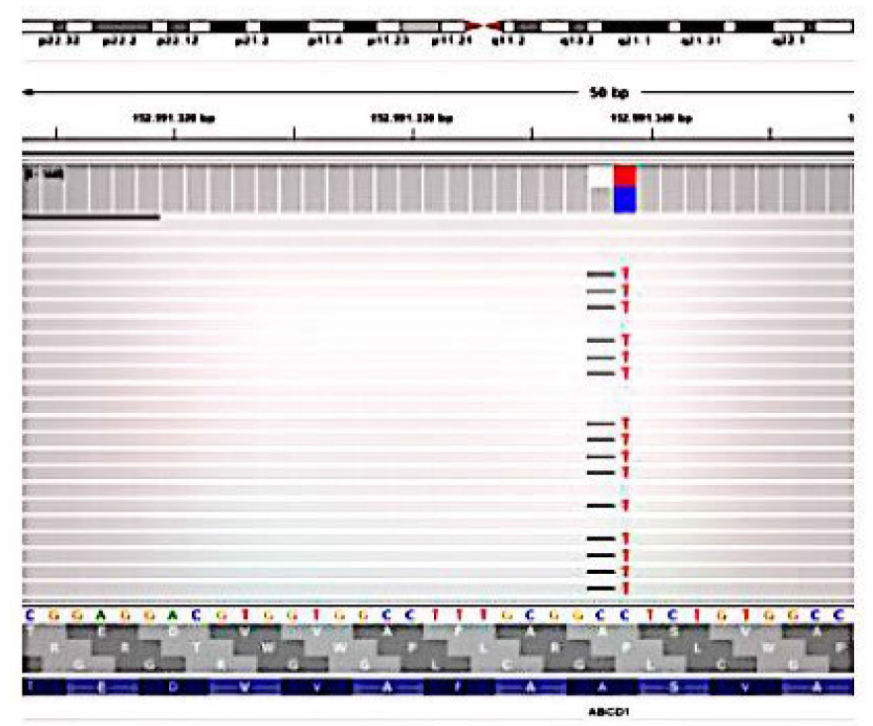

(A)

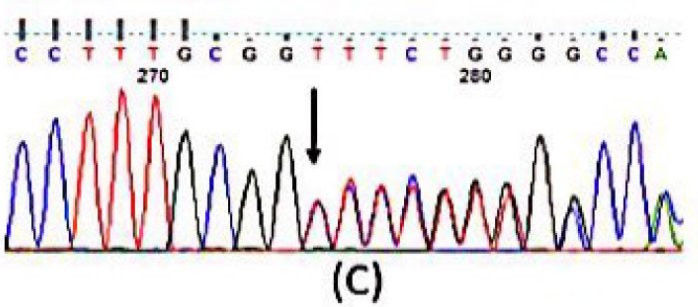

\section{Reference protein}

1 rPVLSAPAPA RONTLKaTAV LLALAAYGAH KVYPLVRCCL APABOLQAPA OEPTQEASOV 61 LAAXAGWRV FLQRLLLILR LLFPAVLCRE TGLLALHSAA IVSATFLSW VARLOGRLAR 121 CIVRKOPRAF OWQLLQULLI ALPATFVNSA IRYLEGQLAL SFRSRLYAHA YRLYFSCQTY

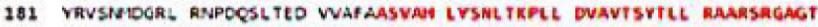

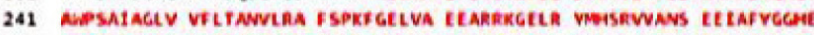

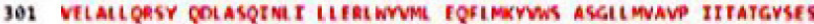

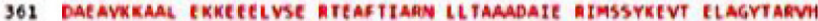

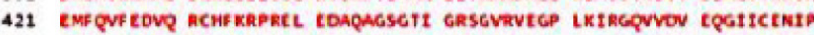

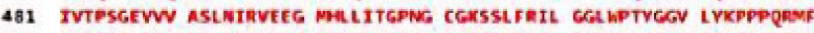

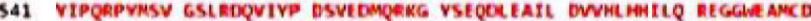

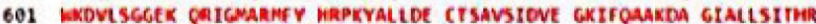

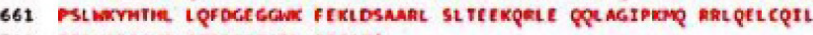
721 GEAVAPAHVP APSPQQGFGGL PCAST*

\section{Protein predicted from variant coding sequence}

1 MPVLSRPRPN AGNTLKRTAY LLALAAYGAH KNPLVRCCL APARGLQAFA GEPTOEASGV 61 AAMCAGNAV FLQRLLWLLR LLFPRVLCRE TGLLALHSAA LVSRTFLSVY VARLOGRLAR 121 CIVRKOPRAF CWQLLQILLI ALPATFVISA IRYLEGQLAL SFRSRLYAHA YRLYFSQQTY 131 VRVSNIDGAL RNPECSLTED WAFAVLIPT STPT*

\section{(B)}

Fig.1:(a) Snapshot of IGV (Integrative Genomics Viewer) showing delins variant chrX:152991338 CC $>$ T; (b) Mutalyzer showing shortened truncated protein Ala206ValfsTer10 as compared with reference protein; (c) Sequencing chromatogram of part of exon 1 in the ABCD1 gene showing frameshift variant. 


\section{Discussion}

X-linked adrenoleukodystrophy has mutations in ABCD1 gene that codes for a protein named ALDP (adrenoleukodystrophy protein). This protein is responsible for transporting very long chain fatty acids (VLCFAs) into the peroxisomes of the cells. So, its malfunction results in the accumulation of VLCFAs in different tissues and organs including myelin, spinal cord, peripheral nerves, adrenal cortex and leydig cells of testis [4]. Two main phenotypes are cerebral ALD and adrenomyeloneuropathy (AMN) [1]. Cerebral demyelinating form is most common during childhood (childhood cerebral ALD: CCALD) and adolescence (adolescent cerebral ALD: ACALD). It is characterized by a rapidly progressive inflammatory demyelination. As a result of severe cognitive and neurologic disability, a vegetative state is reached 2-5 years after onset of symptoms and death thereafter. On the other hand, AMN presents as slowly progressive axonopathy with average age of onset between 20 and 30 years of age in affected males and between 40 and 50 years of age in females. Less frequently, isolated adrenocortical insufficiency (Addison's disease) and asymptomatic type may occur [3]. Management is multidisciplinary; mostly supportive with active physical training and rehabilitation in advanced cases. Hematopoietic stem cell transplantation can arrest cerebral demyelination. It is helpful if done at very early stages when minimal neurological features are present and mortality risk is high. Gene therapy with Lenti D lentiviral vectors are currently under trials $[5,6]$.

With advent of next generation sequencing, it is now possible to identify the mutation causing the disease in families from different ethnic groups. In Iran, Emamalizadeh B et al. found three novel mutations including c.1797dupT, c.879delc and c. $1218 \mathrm{C}>\mathrm{G}$ in the ABCD1 gene [2]. From Delhi, India, Kumar $\mathrm{N}$ et al. identified 4 novel mutations in the ABCD1 gene. The 2 missense novel mutations were c.1673T $>$ C and c.1979G $>A$ in exons 7 and 9 respectively. The 2 deletion/insertion variants
c.1903_04insCCA and c.1993_95delinsGAG were present in exon 9 and 10 respectively [7].

\section{Conclusion}

In this study, we identified a novel frameshift delins variant which is not described earlier. The confirmation of molecular diagnosis is important to correlate genotype with phenotype. Our result will further add on by expanding the mutation spectrum; as well as provide more information for prenatal diagnosis and better patient counselling.

Contributors: CC: drafting the work and revising it critically for important intellectual content; IP: initial motivation and final approval of the version to be published; PS: substantial contributions to the conception or design of the work; or the acquisition, analysis, or interpretation of data for the work. PS will act as a study guarantor. All authors approved the final version of the manuscript and are responsible for all aspects of the study.

Funding: None; Competing interests: None stated.

\section{References}

1. Berger J, Forss-Petter S, Eichler FS. Pathophysiology of X-linked adrenoleukodystrophy. Biochimie. 2014;98:135-142.

2. Emamalizadeh B, Daneshmandpour Y, Tafakhori A, Ranji-Buracaloo S, Shafiee S, Ghods E, et al. Novel ABCD1 gene mutations in Iranian pedigrees with X-linked adrenoleukodystrophy. JPEM. 2019;32:12071215.

3. Engelen M, Kemp S, de Visser M, M Van Geel $\mathrm{B}$, JA Wander R, Aubourg $\mathrm{P}$, et al. X-linked adrenoleukodystrophy (X-ALD): clinical presentation and guidelines for diagnosis, follow-up and management. Orphanet J Rare Dis. 2012;7:51.

4. Kemp S, Berger J, Aubourg P. X-linked adrenoleukodystrophy: clinical, metabolic, genetic and pathophysiological aspects. Biochim Biophys Acta. 2012;1822:1465-1474.

5. Cartier N, Hacein-Bey-Abina S, Bartholomae CC, Veres G, Schmidt M, Kutschera I, et al. Hematopoietic stem cell gene therapy with a lentiviral vector in X-linked adrenoleukodystrophy. Science. 2009;326:818-823.

6. Eichler F, Duncan C, Musolino PL, De Oliveira S, Armant M, Dansereau C, et al. Hematopoietic stem-cell gene therapy for cerebral adrenoleukodystrophy. NEJM. 2017;377:1630-1638.

7. Kumar N, Taneja KK, Kalra V, Behari M, Aneja S, Bansal SK. Genomic profiling identifies novel mutations and SNPs in ABCD1 gene: A molecular, biochemical and clinical analysis of X-ALD cases in India. PLoS One. 2011;6:e25094. 